\title{
Analysis and Optimization of Damping Properties of Constrained Layer Damping Structures with Multilayers
}

\author{
Hongyun Wang $\mathbb{D D}^{1}{ }^{1}$ Heow Pueh Lee $\mathbb{D D}^{2},{ }^{2}$ and Canyi Du ${ }^{1}{ }^{1}$ \\ ${ }^{1}$ School of Automobile and Transportation Engineering, Guangdong Polytechnic Normal University, Guangzhou, \\ Guangdong 510665, China \\ ${ }^{2}$ Department of Mechanical Engineering, National University of Singapore, 9 Engineering Drive 1, Singapore 117575, Singapore
}

Correspondence should be addressed to Canyi Du; ducanyi@gpnu.edu.cn

Received 16 April 2021; Revised 30 October 2021; Accepted 1 November 2021; Published 26 November 2021

Academic Editor: Chao-Ping Zang

Copyright (c) 2021 Hongyun Wang et al. This is an open access article distributed under the Creative Commons Attribution License, which permits unrestricted use, distribution, and reproduction in any medium, provided the original work is properly cited.

\begin{abstract}
Constrained layer damping (CLD) structures, which are one of the composite structures with a softer viscoelastic material (VEM) layer sandwiched between a elastic base layer and a relatively stiffer constraining layer, are widely used in engineering applications for reducing vibration and noise radiation. To accurately predict and effectively control vibration and properly and quickly determine the design parameters, optimal designs for the CLD structures are necessary. The optimal designs depend on thoroughly understanding the damping characteristics of the CLD structures. In addition, for some cases, CLD structures with multi-constrained VEM layers are needed to suppress vibration more effectively. In this paper, an effective modeling method to accurately describe the damping properties and a quick optimization design method using COMSOL were proposed for CLD structures with multilayers in detail. The effects of nondimensional thickness ratios of the VEM and constraining layer to the base layer on the damping properties of CLD structures were analyzed. For CLD structures with different configurations, different constraints were selected to obtain the maximized damping in the optimization design. The conclusions from this research provide an insight into the effects of thicknesses of VEM and constraining layers on the damping properties of CLD structures regardless of its size. The modeling and optimal methods using COMSOL in this paper are not limited to CLD structures and can be used by other structures also.
\end{abstract}

\section{Introduction}

Vibration is undesirable because of the effects of wasting energy, creating unpleasant motions and dynamic stresses, and causing irritating rattles, which will possibly lead to degraded performance, fatigue, and failure of structures or machines. Controlling undesirable vibrations is important in many applications. The constrained layer damping (CLD) structures where a softer viscoelastic material (VEM) layer is constrained between an elastic base structure (base layer) and a relatively stiffer elastic layer (constraining layer) have been demonstrated to have reasonable efficiency in vibration control [1-5]. For the CLD structures, the damping results mainly from shearing in the VEM layer due to the difference between the in-plane displacement of the elastic layers and the low stiffness of the VEM layer [6].
Kerwin [7] was one of the first to analyze the damping effectiveness of CLD using a complex modulus representing the VEM and concluded that the loss factors would depend on the frequency, temperature, and thickness of the constraining layer. Darrouj and Faulkner [8] experimentally verified that the damping would be increased by reducing the thickness of the VEM layer for the reason that the shear energy would be concentrated into a small volume, into which the material would be less able to dissipate compared to larger volume geometries. Using the finite element method, Kosmatka and Liguore [9] revealed that increasing the thickness of the VEM core would generally reduce the natural frequency of the added core mass more than offsetting the increase in the bending stiffness. The loss factor would increase with the core thickness up to an optimal value before decreasing, where the optimal core thickness 
would be dependent on the structure (material and geometry) configuration. Bai and Sun [10] developed a new sandwich theory of vibration damping, which eliminated both perfect interface and constant transverse deformation assumptions. Their results demonstrated that there was an optimal viscoelastic layer stiffness for which the maximum sandwich structural damping would be achieved. The effects of material, temperature, frequency, and dimensions of the base layer on the vibration damping characteristics were discussed by Teng and $\mathrm{Hu}$ [2]. Their results showed that the elastic modulus would decrease at high temperature with a decreasing frequency, and the loss factor would become larger when the thickness of the VEM layer was increased. Moreover, the base layer with higher damping properties would have a more efficient laminate damping than the ones with low damping. Reported studies [8] also showed that the natural frequencies of all modes would decrease with increased thickness of the VEM layer. However, the loss factor would be increased when the thickness ratio of the VEM layer with the base layer exceeded a certain value. The experimental results [11] revealed that to increase the damping capacity of the CLD structure, the VEM layer thickness should be increased, but it should be kept in mind that the mechanical durability of the thick VEM layer would turn out to be insufficient. Another reported study [12] showed that the VEM layer thickness had greater influences on the loss factors than on the storage modulus, and the influences were more pronounced at high frequencies. The bending stiffness of the CLD structure would increase with the VEM thickness even if the storage modulus was reduced. Therefore, the VEM thickness would determine the vibration response of thin sandwich structures. Du et al. [13] pointed out that the high damping of the VEM layer would have a benefit to eliminate the lower order modal resonances for a small plate in the low-frequency range. A theoretical model about fiber metal laminated thin plate with partial CLD patch treatment was established [14]. Based on the calculated results using the model and experimental results, the conclusion was drawn that the larger the patches areas, the closer the treating positions were approaching to the clamped edge, the greater the damping effect would be. From these reported results, it could be concluded that the materials and geometrical characteristics of both VEM and elastic layer have significant effects on the properties of the CLD structures. However, there are some deviations in those results due to the dimensional differences of the base layer.

The desire to maximize the damping properties or to minimize the weight of the CLD structures have led to the studies of optimization by determining the optimal material and geometric parameters of each layer. Lall et al. [15] conducted an optimal design to maximize the modal loss factors and minimize the displacement response with various constraints on the design variables and other requirements such as mass, static stiffness ratio, etc. Lifshitz and Leibowitz [16] summarized earlier optimal design studies about CLD structures and performed an optimal design for maximum damping with a large variety of inequality constraints using a sixth-order motion equation. The constraints were given by normalizing the appropriate parameters with respect to those of an equivalent homogeneous beam, and included weight, rigidity, height of the structure, the thickness of the elastic and VEM layer, and so on. In Hao and Mohan's study [17], maximizing the system loss factor and minimizing the system weight were two main objectives that were combined into the objective function using the weighting method. Constraints were imposed to make the system satisfy the configuration and natural frequency requirements. Madira et al. [18] addressed an optimal design with the objectives of simultaneously minimizing weight and material cost and maximizing the modal damping. The design variables were the number of layers, the layer constituent materials, the orientation angles, and the VEM layer thickness. The topology optimization method was also implemented to achieve the optimal configuration of the damping material of CLD structures [19]. A hierarchical optimization strategy was proposed to optimally design the VEM layer for minimizing the sound radiation power in the literature [20]. However, those optimal designs usually consume a large amount of computational time [21].

The finite element software such as ANSYS, NASTRAN, COMSOL, and LUSAS is a very efficient tool for solving complex problems in the field of design engineering and has been employed to analyze the dynamic and static characteristics of CLD structures [1, 2, 5, 19, 22-24]. However, the optimal designs of CLD structures using finite element software are few.

For some cases, the damping characteristics of CLD structures with one constrained VEM layer which is composed of one VEM layer and one constraining layer on the VEM layer are not substantially effective to suppress the vibration. Alam and Asnani [25], Khatri and Assnani [26], and Khatri [27] pointed out that there would be an increase in the loss factor of CLD structures with the increased number of constrained VEM layers. Considering both material and geometric nonlinearities, an analytical model of fiber metal laminated plates with multiple constrained CLD layers was proposed, and the effects of material properties on nonlinear vibration behaviors were evaluated [28, 29]. However, few reported studies focus on the characteristics of those CLD structures with multiconstrained VEM layers.

From the literature review, it can be found that the damping properties and optimization design for CLD structures have been carried out. However, most research results have deviations due to the different dimensions of CLD structures, and optimization designs usually consume a large amount of computational time. Besides, little work has been conducted considering CLD structures with multilayers. Therefore, aiming at filling this knowledge gap, an effective modeling method to accurately describe the damping properties and quick optimization design method to determine structural parameters using COMSOL were proposed in the present work for CLD structures with multilayers. First, a finite element model of the CLD structure was formulated using COMSOL software, and the modeling method validations were performed by comparing the natural frequencies and loss factors with those obtained by Soni [1]. Thereafter, using the COMSOL model, the 
nondimensional thickness ratios of the VEM and constraining layer to the base layer were considered herein as the variable parameters, and the effects on the damping of CLD structures were compared and analyzed among three CLD structures with different configurations and dimensions. The nondimensional parameters could provide general design guidelines that might be followed by designers, regardless of the dimensions of base structures. Finally, optimization methods were explored to quickly determine the thickness of the VEM and constraining layer by maximizing damping with different constraints for different CLD structures with multiconstrained VEM layers using COMSOL. The conclusions from this research provide an insight into the effects of thicknesses of VEM and constraining layers on the damping properties of CLD structures regardless of its size, and the modeling and optimization design methods using COMSOL proposed here could be easily extended and applied to other types of composite beams.

\section{Formulation and Validation of the Model of the CLD Structure}

2.1. Model Formulation. COMSOL is an advanced numerical software based on the finite element method, which has a friendly predesigned physics interface, and can solve physical problems with numerical models. In the following, the models are constructed and the damping properties are studied for CLD structures using COMSOL.

Figure 1 depicts a typical CLD structure with one constrained VEM layer, where the thicknesses of the base, VEM, and constraining layer are $h_{\mathrm{b}}, h_{\mathrm{v}}$, and $h_{\mathrm{c}}$, respectively.

To formulate the finite element model of CLD structures in COMSOL, the following assumptions are made: there is no slip on the interfaces between layers; only the VEM layer carries transverse shear; the elasticity material and VEM are assumed to be nearly incompressible throughout the thickness; and the material of each layer is considered isotropic and homogeneous.

Three solid mechanics blocks are employed to model the CLD structure. One end of the model is fixed and the other end is made to move freely, namely, clamped-free boundary condition is considered.

In general, the VEMs are frequency- and temperaturedependent. However, if the modulus of VEM does not change too much in the whole frequency domain or changes slightly in a special frequency band, the modulus can be deemed as a constant to describe the VEM approximately or in this special frequency band [30]. In the following studies, the complex constant modulus of the VEM is used. Young's modulus $E_{\mathrm{v}}$ and shear modulus $G_{\mathrm{v}}$ of the VEM are expressed as follows in the complex form:

$$
\begin{aligned}
& E_{\mathrm{v}}=E_{0}\left(1+i \eta_{\mathrm{v}}\right), \\
& G_{\mathrm{v}}=\frac{E_{\mathrm{v}}}{\left(2\left(1+\mathrm{v}_{\mathrm{v}}\right)\right)},
\end{aligned}
$$

where the storage modulus $E_{0}$ is related to the delayed elasticity; $\eta_{\mathrm{v}}$ and $\nu_{\mathrm{v}}$ are the loss factor and Poisson's ratio of the VEM, respectively. $\eta_{\mathrm{v}}$ is the measure of the capacity of VEM to dissipate energy.

For the elastic modulus of the VEM being assumed to be a complex form, the stiffness matrix for the CLD structure is in a complex form as well. This in turn would result in complex eigenvalues. The nonlinear complex eigenvalue problem of equation takes the form

$$
\left(\mathrm{K}-\lambda_{i}^{*} \mathrm{M}\right) \mathrm{U}_{i}=0,
$$

where $\mathrm{M}$ and $\mathrm{K}$ are the mass and complex stiffness matrices, respectively; $U_{i}$ and $\lambda_{i}^{*}$ are the complex eigenvector and associated complex eigenvalue, respectively; the natural frequency $f_{i}$ and the corresponding loss factor $\eta_{i}$ of the $i$ th mode can be calculated as follows [30]:

$$
\begin{aligned}
& f_{i}=\frac{\sqrt{\operatorname{Re}\left(\lambda_{i}^{*}\right)}}{2 \pi}, \\
& \eta_{i}=\frac{\operatorname{Im}\left(\lambda_{i}^{*}\right)}{\operatorname{Re}\left(\lambda_{i}^{*}\right)},
\end{aligned}
$$

where $\eta_{i}$ is used to assess the damping property of CLD structures at mode $i . f_{i}$ and $\eta_{i}$ can easily be obtained using COMSOL.

2.2. Model Validation. To evaluate the accuracy of the formulated COMSOL model of the CLD structure, the natural frequencies and the associated loss factors for the first six modes of an example are calculated using COMSOL. The material and geometrical properties of the example CLD structure are used as the same as those in the literature $[1,30]$ (as shown in Table 1). The CLD structure with the parameters in Table 1 is named the original structure. For the original structure, the constraining layer is assumed to be made from the same material and has the same thickness as that of the base layer. Therefore, the original structure has a better damping efficiency [2].

User-controlled mesh is chosen, and the mapped and swept are applied. For the CLD structure, the damping mostly comes from the shear in the VEM layer, the meshes inside the VEM should be made to be finer to ensure a good representation of the shear behavior. However, for the CLD structure, the thickness of the VEM layer is thinner, the further finer mesh inside the VEM has no effect on the calculated result, and only one layer mesh is applied. Figure 2 represents the meshed image of the designed model in COMSOL.

The calculated results are provided in Table 2 to compare with those results obtained by Soni [1] with the VEM's loss factor of 0.6. The relative errors are also given in Table 2 .

The results obtained using COMSOL are closer to those obtained by Soni [1], and the maximum errors of the natural frequency of $7.48 \%$ and loss factor of $-6.85 \%$ are all acceptable for engineering applications. These results provide validation of the present modeling method using COMSOL.

The reason that the first natural frequency deviation is too large may lie in the following aspects: ignorance of the adhesive interface, interfacial friction and relative slip 


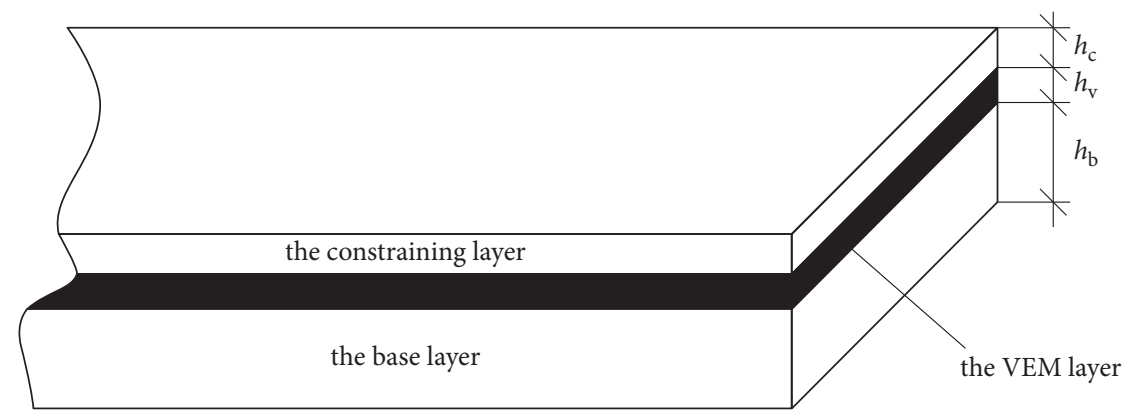

Figure 1: CLD structure with one constrained VEM layer.

TABLe 1: Material and geometrical properties of the CLD structure.

\begin{tabular}{lcc}
\hline & Young's modulus & $E_{b}=E_{c}=6.9 \times 10^{10}\left(\mathrm{~N} \cdot \mathrm{m}^{-2}\right)$ \\
$v_{b}=v_{c}=0.3$ & $\rho_{b}=\rho_{c}=2766\left(\mathrm{~kg} \cdot \mathrm{m}^{-3}\right)$ \\
Base and constraining layer & Poisson's ratio & $h_{b}=h_{c}=1.524 \mathrm{~mm}$ \\
\hline & Density & $E_{0}=1.794 \times 10^{6}\left(\mathrm{~N} \cdot \mathrm{m}^{-2}\right)$ \\
& Thickness & $v_{v}=0.3$ \\
$\eta_{v}=0.6$ & $\rho_{v}=968.1\left(\mathrm{~kg} \cdot \mathrm{m}^{-3}\right)$ \\
VEM layer & Young's modulus & $h_{v}=0.127 \mathrm{~mm}$ \\
& Poisson's ratio & $L=177.8 \mathrm{~mm}$ \\
Structure size & Loss factor & Density \\
& Thickness & $b=12.7 \mathrm{~mm}$ \\
\hline
\end{tabular}

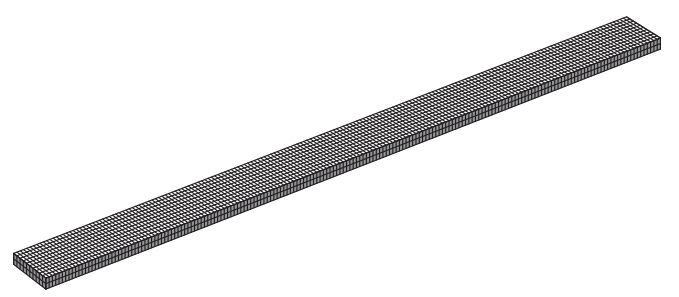

(a)

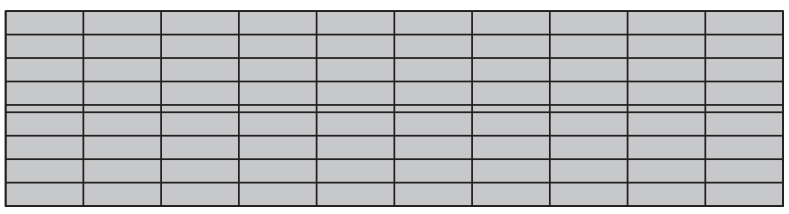

(b)

Figure 2: The meshed image of the CLD structure model in COMSOL. (a) The meshed image. (b) The cross-sectional view.

TABLE 2: The comparison of the natural frequencies and associated loss factors of the first six modes.

\begin{tabular}{|c|c|c|c|c|c|c|}
\hline \multirow{2}{*}{ Modes } & \multicolumn{3}{|c|}{$f_{i}$} & \multicolumn{3}{|c|}{$\eta_{i}$} \\
\hline & Analytical method [1] $(\mathrm{Hz})$ & Present $(\mathrm{Hz})$ & Error (\%) & Analytical method [1] (-) & Present (-) & Error (\%) \\
\hline 1 & 65.5 & 70.4 & 7.48 & 0.1476 & 0.1468 & -0.54 \\
\hline 2 & 298.9 & 308.7 & 3.28 & 0.1392 & 0.1440 & 3.45 \\
\hline 3 & 745.5 & 764.0 & 2.48 & 0.0912 & 0.0929 & 1.86 \\
\hline 4 & 1394.9 & 1437.9 & 3.08 & 0.0528 & 0.0514 & -2.65 \\
\hline 5 & 2261.7 & 2340.6 & 3.49 & 0.0342 & 0.0325 & -4.97 \\
\hline 6 & 3344.0 & 3467.1 & 3.68 & 0.0234 & 0.0218 & -6.84 \\
\hline
\end{tabular}

between each layer, and without consideration of material nonlinearity in modeling using COMSOL, which have more significant impact on the first resonant response.

2.3. Parametric Studies. To understand the role of the thickness of the VEM and constraining layer in the damping properties, three CLD structures are constructed using the validated modeling method. Structure 1 (as shown in Figure 1) has one constrained VEM layer. Structure 2 has two constrained VEM layers, which are symmetrically arranged on both sides of the base layer (as shown in Figure 3), and all constraining layers have the same dimensions, and similarly for all VEM layers. Structure 3 also has one constrained VEM layer, but the thicknesses of the VEM and constraining layer, respectively, are equal to the sum of those of the 


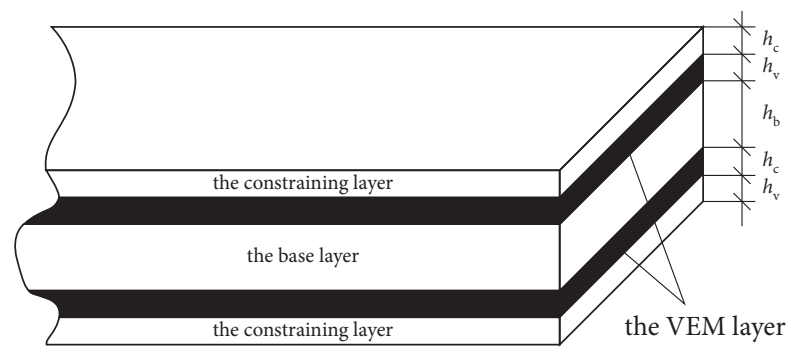

Figure 3: CLD structure with two constrained VEM layers.

corresponding layers of structure 2. Thus, structure 2 and the corresponding structure 3 have the same weight to study the effect of the arrangements on the damping properties of CLD structures at the same weight. The material properties and geometrical dimensions of these three structures are the same as those in Table 1.

For the nondimensional parameters being more suitable for engineering applications and providing a design guideline regardless of the dimension of the base layer, the nondimensional thickness ratios of VEM and constraining layer to the base layer $h_{\mathrm{v}} / h_{\mathrm{b}}, h_{\mathrm{c}} / h_{\mathrm{b}}$ are used.

Considering those technically achievable thickness limits and that the total weight of the CLD structure should not be too large, the thickness ratios are selected to vary from 0.03 to 1.03 .

The studies about the effects of the nondimensional parametric on the damping properties of the CLD structure are achieved using the parametric sweep study in COMSOL.

\section{Results and Analysis of the Eigenfrequency Study}

Figure 4 shows the results of the first six modal loss factors versus $h_{\mathrm{c}} / h_{\mathrm{b}}$ and $h_{\mathrm{v}} / h_{\mathrm{b}}$ of structure 1 . It is displayed that the higher the modes, the smaller the impacts of the $h_{\mathrm{c}} / h_{\mathrm{b}}$ and $h_{\mathrm{v}} / h_{\mathrm{b}}$ on the loss factors are. It is also concluded that the first six modal loss factors are directly proportional to $h_{\mathrm{c}} / h_{\mathrm{b}}$, when $h_{\mathrm{c}} / h_{\mathrm{b}}$ is below about 0.7 . Thereafter, the modal loss factors start to drop. The loss factor of the first mode is directly proportional to $h_{\mathrm{v}} / h_{\mathrm{b}}$, and the others are inversely proportional to $h_{\mathrm{v}} / h_{\mathrm{b}}$. When $h_{\mathrm{c}} / h_{\mathrm{b}}$ is below 0.4 , or $h_{\mathrm{v}} / h_{\mathrm{b}}$ is below 0.3 , the first three modal loss factors sharply change, and then steadily change.

Figure 5 shows the results of the first six modal frequencies versus $h_{\mathrm{c}} / h_{\mathrm{b}}$ and $h_{\mathrm{v}} / h_{\mathrm{b}}$ of structure 1 . It is shown that the first six loss factors are inversely proportional to $h_{\mathrm{c}} / h_{\mathrm{b}}$, when $h_{\mathrm{c}} / h_{\mathrm{b}}$ is below about 0.5 . Thereafter, the modal loss factors start to rise. The first six loss factors are inversely proportional to $h_{\mathrm{v}} / h_{\mathrm{b}}$. It is also displayed that the first three modal frequencies are below $1000 \mathrm{~Hz}$.

For the fact that the low-frequency domain is of more interest in practice, and the effects of $h_{\mathrm{c}} / h_{\mathrm{b}}$ and $h_{\mathrm{v}} / h_{\mathrm{b}}$ on the higher loss factors are small, only the first three modal loss factors and natural frequencies are considered in the following studies.

Figure 6 shows the variation in the first three modal loss factors with the ratios $h_{\mathrm{c}} / h_{\mathrm{b}}$ and $h_{\mathrm{v}} / h_{\mathrm{b}}$.
It can be seen in Figure 6 that $\eta_{i}$ increases initially, reaches its maximum, and then decreases with the increasing $h_{\mathrm{c}} / h_{\mathrm{b}}$ at a constant $h_{\mathrm{v}} / h_{\mathrm{b}}$. After $h_{\mathrm{c}} / h_{\mathrm{b}}>1$ and $h_{\mathrm{v}} / h_{\mathrm{b}}>1$, with a further increase of $h_{\mathrm{c}} / h_{\mathrm{b}}, \eta_{3}$ increases again after obtaining its minimum value (as shown in Figure 6(i)).

For CLD structures, the damping comes from two parts mainly. One is the damping of the VEM layer, and the other is the constrained damping, which comes from the constraint of the constraining layer on the motion of the VEM layer [3].

It can be concluded from the above results that the increase in the thickness of the constraining layer would increase the constrained damping of CLD structures with the increased ability to restrain the motion of the VEM layer. However, when the thickness of the constraining layer exceeds a certain value, the stiffness of the CLD structure would increase more than damping, which makes the CLD structure quite stiff and the shear deformation in the VEM layer reduced, and the VEM would no longer have sufficient flexibility to dissipate as much energy, and the loss factors of CLD structure would decrease.

It is also interesting to note in Figure 6 that $\eta_{1}$ always increases with increasing $h_{\mathrm{v}} / h_{\mathrm{b}}$. This behavior is less observed at higher modes. For the second mode and the third mode, the loss factors sharply decrease at the beginning, then marginally increase with increasing $h_{\mathrm{v}} / h_{\mathrm{b}}$. These results illustrate that the damping coming from the VEM is more effective for the first mode. However, for the other modes, the increase in $h_{\mathrm{v}} / h_{\mathrm{b}}$ contributes more to the added VEM's ability to resist the constraint of the constraining layer than to the added damping of the structure when the thickness of the VEM layer is too small. When $h_{\mathrm{v}} / h_{\mathrm{b}}>1$, the further increase of $h_{\mathrm{v}} / h_{\mathrm{b}}$ contributes more to the added damping than to the added VEM's ability to resist the constraint of the constraining layer, and $\eta_{2}$ and $\eta_{3}$ increase again.

In Figure 6, it is also shown that the loss factors of structure 2 are always higher than those of structure 1 . When $h_{\mathrm{v}} / h_{\mathrm{b}}>1$ and $h_{\mathrm{c}} / h_{\mathrm{b}}<1$, at the same weight, the first two loss factors of structure 3 are higher than those of structure 2 .

In short, the above results illustrate that different thicknesses and arrangements of the VEM and constraining layer have varying effects on the damping properties of the CLD structures. Better damping properties can be obtained by symmetrically arranging the constrained VEM layers on both sides of the base layer. After obtaining the maximum value with the increase in the constraining layer thickness, the damping properties of the CLD structure will decline sharply 


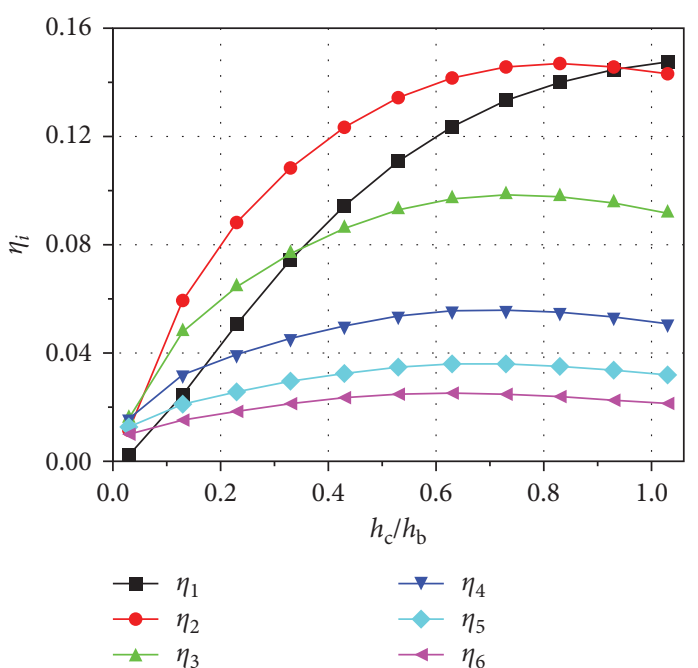

(a)

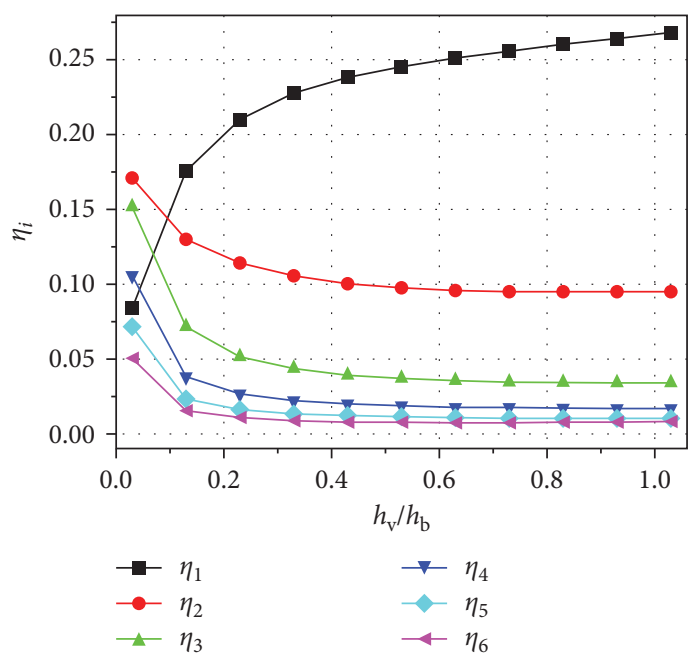

(b)

FiguRE 4: The parameter effects on the first six modal loss factors of structure 1 . (a) $h_{c} / h_{b}$. (b) $h_{v} / h_{b}$.

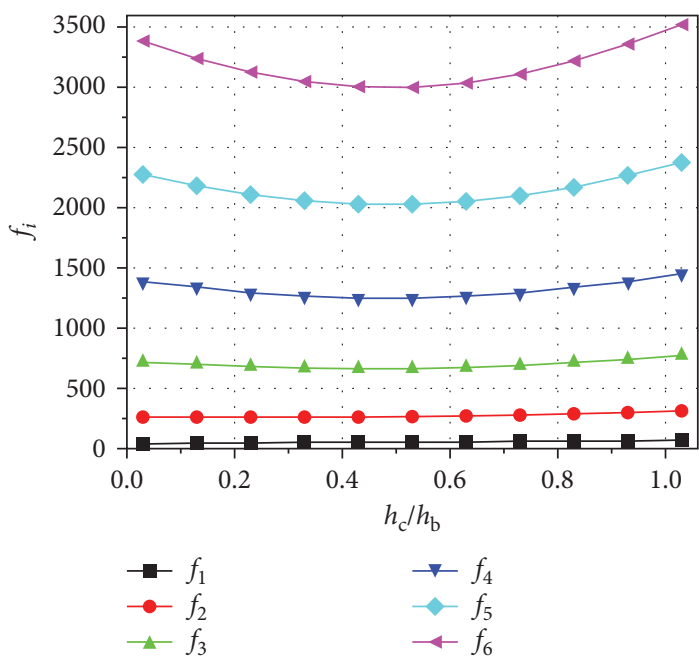

(a)

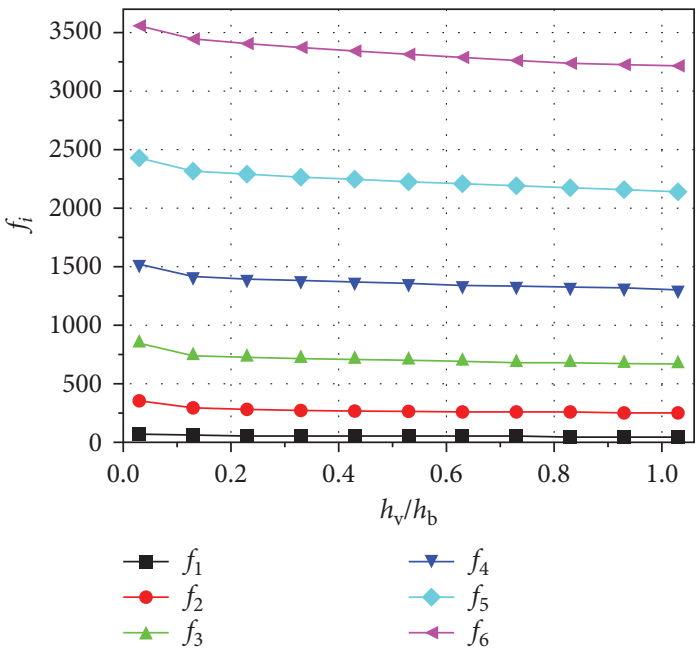

(b)

Figure 5: The parameter effects on the first six modal frequencies of structure 1 . (a) $h_{c} / h_{b}$. (b) $h_{v} / h_{b}$.

when the thickness of the constraining layer exceeds the thickness of the base layer except for the thickness of the VEM layer being thicker and exceeding that of the base layer's. The first modal loss factor always increases with the increase in the VEM layer thickness, but the other modal loss factors would increase only when the VEM layer thickness exceeds a certain value. For the same weight, when the thickness of the VEM exceeds that of the base layer, and the thickness of the constraining layer is very small, the damping property of the CLD structure with one constrained layer is better than that with two constrained layers of symmetrical layout.

Figure 7 shows the variation in the first three modal frequencies with the ratios of $h_{\mathrm{c}} / h_{\mathrm{b}}$ and $h_{\mathrm{v}} / h_{\mathrm{b}}$.

It can be observed in Figure 7 that $f_{i}$ decreases with the increasing $h_{\mathrm{v}} / h_{\mathrm{b}}$ that contributes to the added mass and reduced stiffness of the CLD structures. However, the influence of the increase of $h_{\mathrm{c}} / h_{\mathrm{b}}$ on the stiffness is more obvious than that on the mass of the CLD structures, and $f_{i}$ increases with the increase in $h_{\mathrm{c}} / h_{\mathrm{b}}$ mostly.

From Figure 7, it also can be seen that the natural frequencies of structure 2 are lower than those of structure 3 at the same weight of the CLD structures and are slightly higher than those of structure 1 .

\section{Optimization}

As indicated in the above results, the change in the thickness of the VEM and constraining layer will lead to the change in the damping properties and natural frequencies of CLD structures. To effectively control the vibration, it is required to seek an appropriate thickness of the VEM and constraining layer, which would yield the maximum shear 


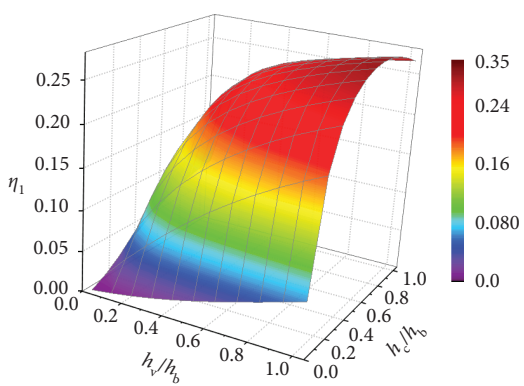

(a)

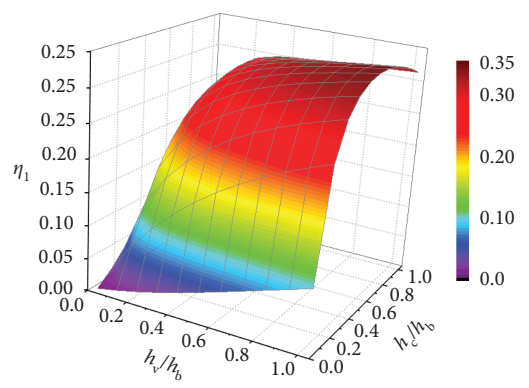

(d)

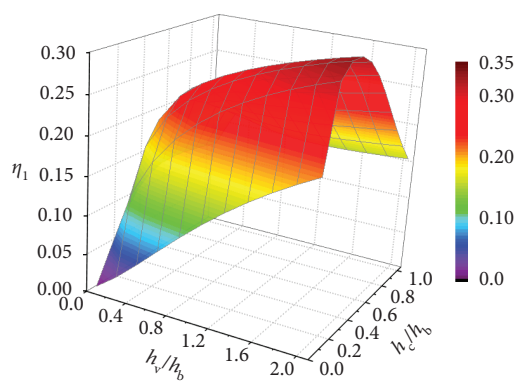

(g)

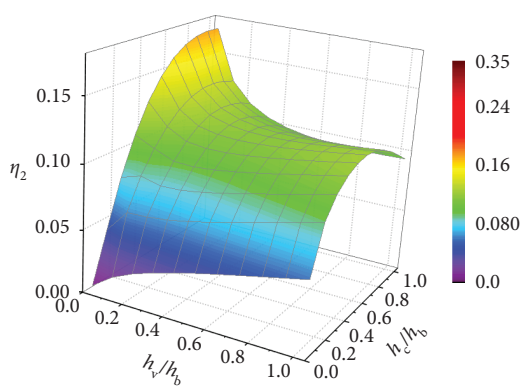

(b)

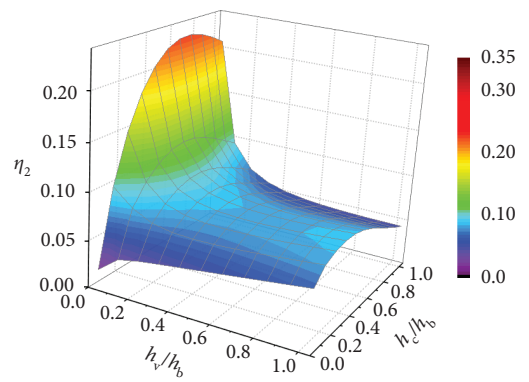

(e)

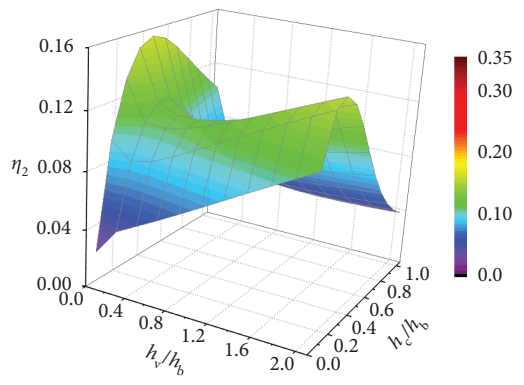

(h)

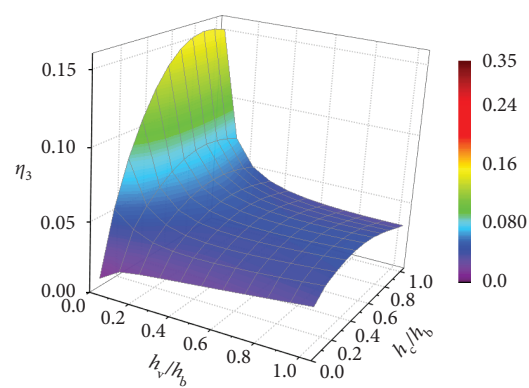

(c)

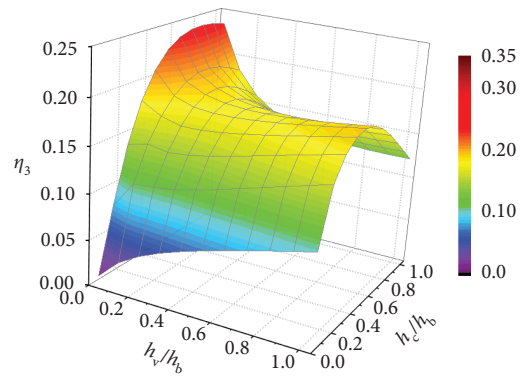

(f)

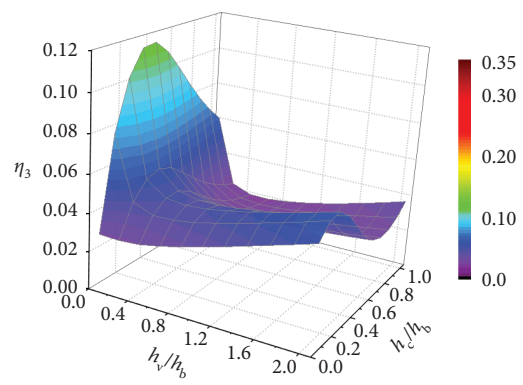

(i)

Figure 6: The parameter effects on the first three modal loss factors. (a) Mode 1 for structure 1. (b) Mode 2 for structure 1. (c) Mode 3 for structure 1. (d) Mode 1 for structure 2. (e) Mode 2 for structure 2. (f) Mode 3 for structure 2. (g) Mode 1 for structure 3. (h) Mode 2 for structure 3. (i) Mode 3 for structure 3.

energy distribution, and thus resulting in the maximum modal loss factor. Here, the optimization problem is formulated for CLD structures with different configurations using COMSOL to achieve maximum damping properties. In the optimization module of COMSOL, the Nelder-Mead method is used, which is a gradient free method. On the optimization interface of COMSOL, the objective function, control variables, and parameters and constrains for the optimization problem can be set easily.

4.1. Optimal Design Formulations. The objectives of the optimal design of the CLD structures usually are to maximize their structural damping, which is given by the modal loss factors, to control the natural frequencies of the modes of interest, or to minimize the total weight and so on.

Consideration of all possible situations is important for an effective CLD structure design. However, multiobjective optimization is overcomplicated and it will lead to too many solutions. Thus, single-objective optimization is employed herein, namely, the only objective function is maximizing the damping of CLD structures.
In addition, the vibration in the low-frequency domain is of most concern in practice, and the sum of the first three modal loss factors is selected as the single-objective function.

The results of the effects of $h_{\mathrm{c}} / h_{\mathrm{b}}$ and $h_{\mathrm{v}} / h_{\mathrm{b}}$ on the sum of the first three loss factors of CLD $\eta$ are shown in Figure 8. For structure 1, the maximum loss factor $\eta$ is about 0.449 when $h_{\mathrm{v}} / h_{\mathrm{b}}$ and $h_{\mathrm{c}} / h_{\mathrm{b}}$ are about 1.03 and 0.63 , respectively, which is higher than 0.384 of the original structure. The total weight is only $97.93 \%$ of the original structure weight. The corresponding first three natural frequencies are $48.71 \mathrm{~Hz}$, $221.83 \mathrm{~Hz}$, and $581.61 \mathrm{~Hz}$, respectively, which are lower than those of the original structure (as shown in Table 2). Lower the natural frequency of the structure, the easier it will be excited; this result is not deemed to be desirable, and the first modal frequency should be controlled for structure 1 .

For structure 2, the maximum value of loss factor $\eta$ is about 0.617 when $h_{\mathrm{v}} / h_{\mathrm{b}}$ and $h_{\mathrm{c}} / h_{\mathrm{b}}$ are about 0.03 and 0.93 , respectively, which is significantly higher than 0.449 of structure 1 . The corresponding first three natural frequencies are $99.39 \mathrm{~Hz}, 389.43 \mathrm{~Hz}$, and $869.27 \mathrm{~Hz}$, respectively, which are higher than those of the original structure (as shown in 


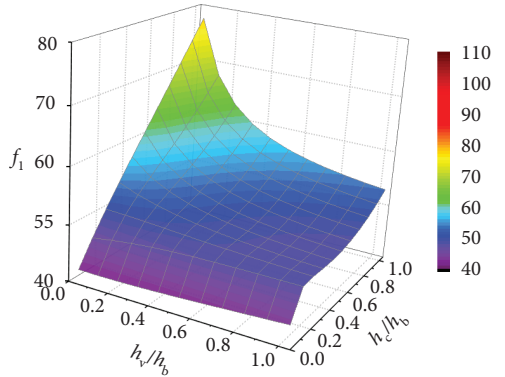

(a)

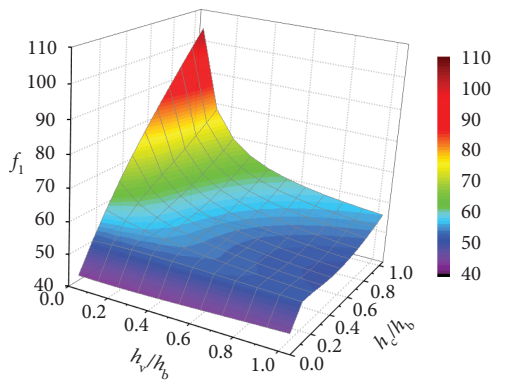

(d)

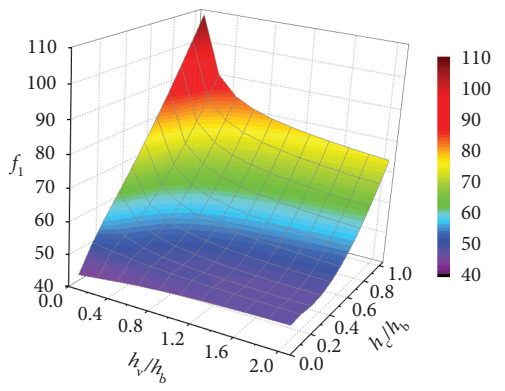

(g)

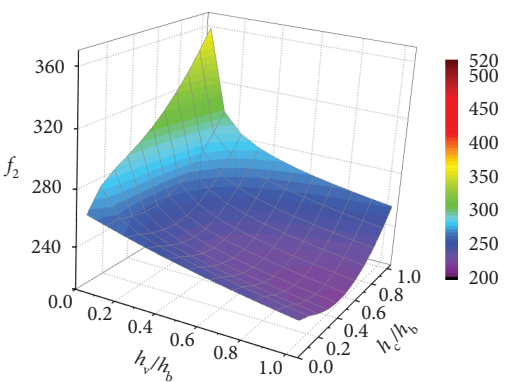

(b)

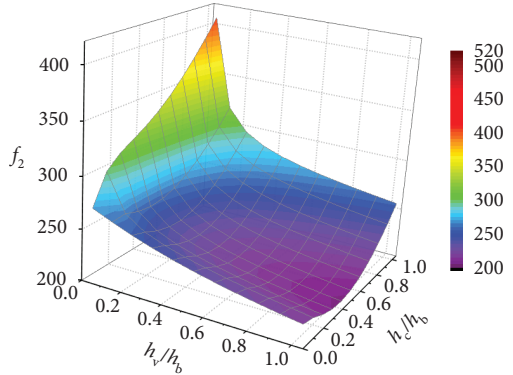

(e)

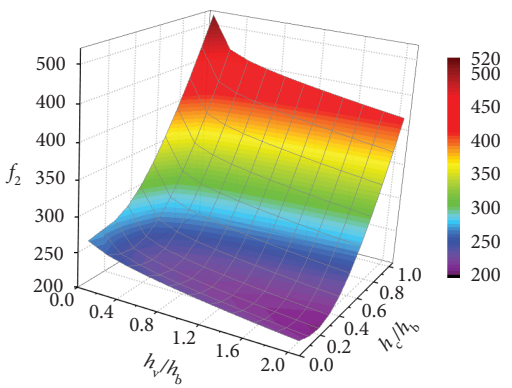

(h)

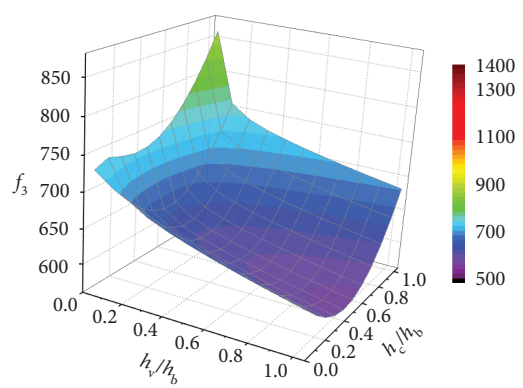

(c)

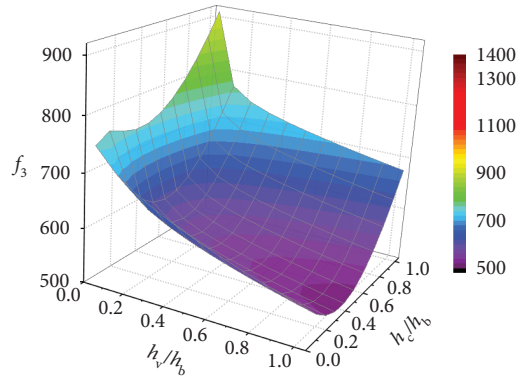

(f)

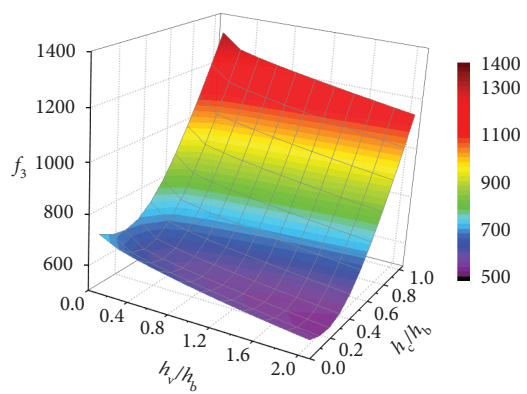

(i)

Figure 7: The parameter effects on the first three modal frequencies. (a) Mode 1 for structure 1. (b) Mode 2 for structure 1 . (c) Mode 3 for structure 1. (d) Mode 1 for structure 2. (e) Mode 2 for structure 2. (f) Mode 3 for structure 2. (g) Mode 1 for structure 3. (h) Mode 2 for structure 3. (i) Mode 3 for structure 3.

Table 2). However, the weight increases by $41.97 \%$ than that of the original structure. From the lightweight point of view, this result is not deemed to be desirable, and the weight of structure 2 should be controlled.

For the above reasons, the natural frequencies of a particular set of modes of interest and the total weight of the
CLD structure are selected as constraint conditions and the nondimensional thickness ratios $h_{\mathrm{c}} / h_{\mathrm{b}}$ and $h_{\mathrm{v}} / h_{\mathrm{b}}$ are selected as design variables, which are allowed to vary from 0.03 to 1.03 .

A general optimization problem for the CLD structure can be stated as

$$
\begin{aligned}
& \text { find } \mathbf{h}=\frac{h_{\mathrm{c}} / h_{\mathrm{b}}}{h_{\mathrm{v}} / h_{\mathrm{b}}}, \\
& \max \quad \eta=\sum_{i=1}^{3} \eta_{i}, \\
& \text { subject to } \quad Q_{k}^{l} \leq Q_{k} \leq Q_{k}^{u} \quad k=1, \ldots, n, \\
& \text { where } \frac{\varepsilon_{\mathrm{c}}^{l} \leq h_{\mathrm{c}}}{h_{\mathrm{b}} \leq \varepsilon_{\mathrm{c}}^{u}}, \frac{\varepsilon_{\mathrm{v}}^{l} \leq h_{\mathrm{v}}}{h_{\mathrm{b}} \leq \varepsilon_{\mathrm{v}}^{u}},
\end{aligned}
$$




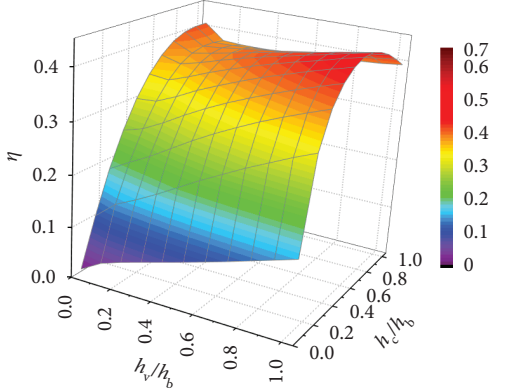

(a)

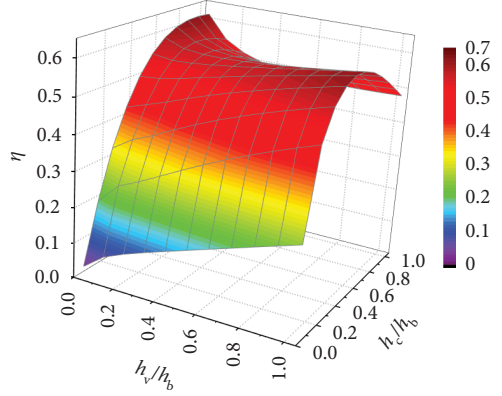

(b)

Figure 8: The parameter effects on the sum of the first three modal loss factors. (a) Structure 1. (b) Structure 2.

TABLE 3: The optimal results.

\begin{tabular}{|c|c|c|c|c|c|c|}
\hline \multirow{3}{*}{\multicolumn{2}{|c|}{ Variables and results }} & \multirow{3}{*}{ Original structure } & \multicolumn{4}{|c|}{ Optimal results } \\
\hline & & & \multicolumn{2}{|c|}{ Structure 1} & \multicolumn{2}{|c|}{ Structure 2} \\
\hline & & & Values & Change rate (-) & Values & Change rate (-) \\
\hline \multirow{2}{*}{\multicolumn{2}{|c|}{$\begin{array}{l}h_{c} / h_{b}(-) \\
h_{v} / h_{b}(-)\end{array}$}} & 1 & 0.97 & $-3 \%$ & 0.49 & $-51 \%$ \\
\hline & & 0.08 & 0.03 & $-62.5 \%$ & 0.03 & -62.5 \\
\hline \multirow{5}{*}{$f_{i}(\mathrm{~Hz})$} & $f_{1}$ & 70.41 & 75.52 & $7.26 \%$ & 75.65 & $7.44 \%$ \\
\hline & $f_{2}$ & 308.73 & 350.41 & $13.50 \%$ & 329.78 & $6.82 \%$ \\
\hline & $f_{3}$ & 763.99 & 836.16 & $9.45 \%$ & 751.34 & $-1.66 \%$ \\
\hline & $\eta_{1}$ & 0.1468 & 0.0830 & $-43.46 \%$ & 0.0995 & $-32.22 \%$ \\
\hline & $\eta_{2}$ & 0.1440 & 0.1711 & $18.82 \%$ & 0.2188 & $51.94 \%$ \\
\hline \multirow{3}{*}{$\eta_{i}(-)$} & $\eta_{3}$ & 0.0929 & 0.1527 & $64.37 \%$ & 0.2141 & $130.46 \%$ \\
\hline & $\sum_{i=1}^{3} \eta_{i}$ & 0.3837 & 0.4068 & $6.02 \%$ & 0.5324 & $38.75 \%$ \\
\hline & & 0.0193 & 0.0093 & $-51.81 \%$ & 0.0190 & $-1.5 \%$ \\
\hline
\end{tabular}

where the loss factor $\eta$ is the objective function, which is the sum of the first three modal loss factors; $\mathbf{h}$ is the vector of design variables; $\varepsilon_{\mathrm{c}}^{l}, \varepsilon_{\mathrm{c}}^{u}, \varepsilon_{\mathrm{v}}^{l}$, and $\varepsilon_{\mathrm{v}}^{u}$, respectively, are the lower and upper limits of the design variables $h_{\mathrm{c}} / h_{\mathrm{b}}$ and $h_{\mathrm{v}} / h_{\mathrm{b}} ; Q_{k}$ is the $k$ th inequality constraint, and its lower and upper limits are $Q_{k}^{\mathrm{l}}$ and $Q_{k}^{\mathrm{u}}$, respectively.

For structure $1, f_{1} \geq 65 \mathrm{~Hz}$ is selected as the constraint condition. For structure $2, f_{1} \geq 65 \mathrm{~Hz}$ and the total weight $W \leq 0.0193 \mathrm{~kg}$ are selected as constraints, where $0.0193 \mathrm{~kg}$ is the weight of the original structure.

4.2. Optimal Results. The optimal results are listed in Table 3, which are compared with those of the original structure.

For structure 1, the optimal damping of the sum of the first three mode loss factors and the natural frequency of the first mode, respectively, are 0.4068 and $75.52 \mathrm{~Hz}$ when $h_{\mathrm{c}} / h_{\mathrm{b}}=0.97$ and $h_{\mathrm{v}} / h_{\mathrm{b}}=0.03$, which are higher than those of the original structure by $6.02 \%$ and $7.26 \%$, respectively, and the weight is reduced by $51.81 \%$.

For structure 2, the optimal damping is 0.5324 when $h_{\mathrm{c}} / h_{\mathrm{b}}=0.49$ and $h_{\mathrm{v}} / h_{\mathrm{b}}=0.03$, which is higher than that of the optimized structure 1 by $30.88 \%$, and that of the original structure by $38.75 \%$. The natural frequency of the first mode is $75.65 \mathrm{~Hz}$, which is higher than that of the original structure by $7.44 \%$, and the weight is reduced by $1.5 \%$ than that of the original structure.

\section{Summary and Conclusions}

In this paper, a modeling method for CLD structures was proposed using COMSOL. Using the dynamic models, the damping properties of CLD structures with multiconstrained VEM layers were studied accurately. Moreover, using COMSOL, a quick optimization design method was provided by maximizing damping with different constraints for different CLD structures with multiconstrained VEM layers.

The following conclusions are drawn:

(1) For a prespecified base structure, when higher damping is desired to control vibration, and at the same time the natural frequencies are not desired to change too much, the method to use two constrained VEM layers which are symmetrically arranged on both sides of the base layer are the best choice.

(2) For the first mode, increasing the VEM thickness means increasing the energy absorption, and increasing the constraining layer thickness increases the constrained shear force in VEM, which increases the damping of the CLD structure. Nevertheless, when the constraining layer reached a certain thickness, the further increase in the constraining layer thickness could cause the decrease in damping. For other modes, increasing the 
VEM thickness means decreasing the energy absorption, and increasing the constraining layer thickness increases the constrained shear force in VEM, which increases the damping of the CLD structure. Nevertheless, when the constraining layer reached a certain thickness, the increase in the constraining layer thickness could cause the decrease of damping.

(3) For the same weight, when the VEM thickness exceeds that of the base layer, and the constraining layer thickness is thinner, the damping property of CLD structures with one constrained VEM layer is better than those with two constrained VEM layers of the symmetrical layout.

(4) Using COMSOL, different objective functions, control variables and parameters, constrains, and so on for the optimization problem are very convenient to set for different CLD structure configurations. In this paper, maximizing the sum of the first three modal loss factors is the objective function, and the thicknesses of the VEM and constraining layer are control parameters. For the CLD structure with one constrained VEM layer, the first modal natural frequency is selected as the constraint. For the CLD structure with symmetrical layout's two constrained VEM layers, the first modal natural frequency and the total weight are selected as constraints.

From the present study, it is revealed that COMSOL software is a very effective tool to study the damping characteristics and perform the optimization design for CLD structures. The present model constructed using COMSOL can predict the damping characteristics accurately and obtain the optimization design quickly. The modeling and optimal methods using COMSOL in this paper are not limited to CLD structures and can be used by other structures also.

\section{Data Availability}

The data used to support the findings of this study are included within the article.

\section{Conflicts of Interest}

The authors declare that they have no conflicts of interest.

\section{Acknowledgments}

This research was supported by the Science and Technology Planning Projects of Guangzhou, P. R. China (201905010007), the Guangdong Province Applied Major Project, P. R. China (2015B090920006), and the Nature Science Foundation of Guangdong Province, P. R. China (2018A030313947).

\section{References}

[1] M. L. Soni, "Finite element analysis of viscoelastically damped sandwich structures," Shock and Vibration, vol. 55, no. 1, pp. 97-109, 1981.
[2] T. L. Teng and N. K. Hu, "Analysis of damping characteristics for viscoelastic laminated beams," Computer Methods in Applied Mechanics and Engineering, vol. 190, pp. 3881-3892, 2001.

[3] M. D. Rao, "Recent applications of viscoelastic damping for noise control in automobiles and commercial airplanes," Journal of Sound and Vibration, vol. 262, pp. 457-474, 2003.

[4] J. S. Grewal, Vibration and thermal Analysis and Optimum Design of Viscoelastic sandwich Beam, Master Thesis, Concordia University, Montreal, Canada, 2011.

[5] A. Shinde and B. P. Ganesh, "Passive viscoelastic constrained layer damping technology for structural application," Journal of Emerging Technologies and Innovative Research, vol. 3, no. 4, pp. 60-68, 2016.

[6] M. Bilasse, E. M. Daya, and L. Azrar, "Linear and nonlinear vibrations analysis of viscoelastic sandwich beams," Journal of Sound and Vibration, vol. 329, pp. 4950-4969, 2010.

[7] E. M. Kerwin, "Damping of flexural waves by a constrained viscoelastic layer," Journal of the Acoustical Society of America, vol. 31, no. 7, pp. 952-962, 1959.

[8] M. N. Darrouj and R. G. Faulkner, "Optimum design of constrained layer damping panels," Materials \& Design, vol. 10, no. 4, pp. 202-208, 1989.

[9] J. B. Kosmatka and S. L. Liguore, "Review of methods for analyzing constrained-layer damped structures," Journal of Aerospace Engineering, vol. 6, no. 3, pp. 268-283, 1993.

[10] J. M. Bai and C. T. Sun, "The effect of viscoelastic adhesive layers on structural damping of sandwich beams," Journal of Structural Mechanics, vol. 23, no. 1, pp. 1-16, 1995.

[11] E. Sarlin, Y. Liu, M. Vippola et al., "Vibration damping properties of steel/rubber/composite hybrid structures," Composite Structures, vol. 94, pp. 3327-3335, 2012.

[12] L. Irazu and M. J. Elejabarrieta, "The influence of viscoelastic film thickness on the dynamic characteristics of thin sandwich structures," Composite Structures, vol. 134, pp. 421-428, 2015.

[13] S. Du, F. An, and B. Liu, "On the sound transmission loss of finite plates with constrained viscoelastic layer," Applied Acoustics, vol. 149, pp. 32-38, 2019.

[14] Z. Xu, H. Li, W.-Yu Wang, and B.-C. Wen, "The analysis of vibration characteristics of fiber metal laminated thin plate with partial constrained layer damping patches treatment," Meccanica, vol. 55, no. 1-3, 2019.

[15] A. K. Lall, B. C. Nakra, and N. T. Asnani, "Optimum design of viscoelastically damped sandwich plates," Engineering Optimization, vol. 6, pp. 197-205, 1983.

[16] J. M Lifshitz and M. Leibowitz, "Optimal sandwich beam design for maximum viscoelastic damping," Internal Journal of Solids Structures, vol. 23, no. 7, pp. 1027-1034, 1987.

[17] M. Hao and D. R. Mohan, "Vibration and damping analysis of a sandwich beam containing a viscoelastic constraining layer," Journal of Composite Materials, vol. 39, no. 18, pp. 1621-1643, 2005.

[18] J. F. A. Madeira, A. L. Araújo, C. M. Mota Soare, C. A. Mota Soares, and A. J. M. Ferreira, "Multiobjective design of viscoelastic laminated composite sandwich panels," Composites Part B, vol. 77, pp. 391-401, 2015.

[19] Y. K. Xu, W. G. Gao, Y. H. Yu et al., "Dynamic optimization of constrained layer damping structure for the headstock of machine tools with modal strain energy method," Shock and Vibration, vol. 2017, Article ID 2736545, 13 pages, 2017.

[20] D. D. Zhang, T. Qi, and Z. Ling, “A hierarchical optimization strategy for position and thickness optimization of 
constrained layer damping/plate to minimize sound radiation power," Advances in Mechanical Engineering, vol. 10, no. 10, pp. 1-15, 2018.

[21] O. Stepan, P. Martin, and D. Tomas, "Case studies on optimization problems in MATLAB and COMSOL Multiphysics by means of the LiveLink," AIP Conference Proceedings, vol. 1738, pp. 370006-1-370006-3, 2016.

[22] Y. P. Lu, J. W. Killian, and G. C. Everstine, "Vibrations of three layered damped sandwich plate composites," Journal of Sound and Vibration, vol. 64, no. 1, pp. 63-71, 1979.

[23] C. D. Johnson and D. A. Kienholz, "Finite element prediction of damping in structures with constrained viscoelastic layers," AIAA Journal, vol. 20, no. 9, pp. 1284-1290, 1981.

[24] B. Kumar, ANSYS and Analytical Modeling of Elastic-Viscoelastic-Elastic sandwich Beams, Master Thesis, Tennessee Technological University, Cookeville, TN, USA, 2010.

[25] N. Alam and N. T. Asnani, "Vibration and damping analysis of multilayered rectangular plates with constrained viscoelastic layers," Journal of Sound and Vibration, vol. 97, no. 4, pp. 597-614, 1984.

[26] K. N. Khatri and N. T. Asnani, "Vibration and damping analysis of multilayered conical shells," Composite Structures, vol. 33, pp. 143-157, 1995.

[27] K. N. Khatri, "Axisymmetric vibration of multilayered conical shells with core layers of viscoelastic material," Computers \& Structures, vol. 58, no. 2, pp. 389-406, 1996.

[28] H. Li, Z. Wang, H. Lv et al., "Nonlinear vibration analysis of fiber reinforced composite cylindrical shells with partial constrained layer damping treatment," Thin-Walled Structures, vol. 157, Article ID 107000, 2020.

[29] H. Li, Z. Li, B. Safaei et al., "Nonlinear vibration analysis of fiber metal laminated plates with multiple viscoelastic layers," Thin-Walled Structures, vol. 168, Article ID 108297, 2021.

[30] S. Ren and G. Zhao, "A four-node quadrilateral element for vibration and damping analysis of sandwich plates with viscoelastic core," Journal of Sandwich Structures and Materials, vol. 21, no. 3, pp. 1072-1118, 2019. 